\title{
Langmuir-Schaefer film deposition onto honeycomb porous films for retinal tissue engineering
}

\author{
Maria Teresa Calejo ${ }^{\mathrm{a}, 1^{*}}$, Tanja Ilmarinen ${ }^{\mathrm{b}, 1}$, Elina Vuorimaa-Laukkanen ${ }^{\mathrm{c}}$, Elina Talvitie ${ }^{\mathrm{a}}$, Hanna M. \\ Hakola $^{\mathrm{c}}$, Heli Skottman ${ }^{\mathrm{b}}$, Minna Kellomäki ${ }^{\mathrm{a}}$
}

${ }^{a}$ Faculty of Biomedical Sciences and Engineering, BioMediTech, Tampere University of Technology, Tampere, Finland

${ }^{\mathrm{b}}$ Faculty of Medicine and Life Sciences, BioMediTech, University of Tampere, Tampere, Finland

${ }^{c}$ Laboratory of Chemistry and Bioengineering, Tampere University of Technology, Tampere, Finland

* Corresponding author. E-mail: teresa.calejo@tut.fi; Tel: +358 503014048 (M. T. Calejo)

${ }^{1}$ These authors equally contributed to this work

\begin{abstract}
Age-related macular degeneration (AMD) is the leading cause of vision loss in senior citizens in the developed world. The disease is characterised by the degeneration of a specific cell layer at the back of the eye - the retinal pigment epithelium (RPE), which is essential in retinal function. The most promising therapeutic option to restore the lost vision is considered to be RPE cell transplantation. This work focuses on the development of biodegradable biomaterials with similar properties to the native Bruch's membrane as carriers for RPE cells. In particular, the breath figure (BF) method was used to create semi-permeable microporous films, which were thereafter used as the substrate for the consecutive Langmuir-Schaefer (LS) deposition of highly organised layers of collagen type I and collagen type IV. The newly developed biomaterials were further characterised in terms of surface porosity, roughness, hydrophilicity, collagen distribution, diffusion properties and hydrolytic stability. Human embryonic stem cell-derived RPE cells (hESC-RPE) cultured on the biomaterials showed good adhesion, spreading and morphology, as well as the expression of specific protein markers. Cell function was additionally confirmed by the assessment of the phagocytic capacity of hESC-RPE. Throughout the study, microporous films consistently showed better results as cell culture materials for
\end{abstract}


hESC-RPE than dip-coated controls. This work demonstrates the potential of the BF-LS combined technologies to create biomimetic prosthetic Bruch's membranes for hESC-RPE transplantation.

\section{Keywords}

Retinal pigment epithelium; pluripotent stem cells; tissue engineering; honeycomb films; LangmuirSchaefer films 


\section{Introduction}

Age-related macular degeneration (AMD) is a neurodegenerative ocular disease and a leading cause of central blindness in developed countries, estimated to affect more than 196 million people worldwide by 2020 [1]. Loss of vision in AMD is primarily concerned with the progressive degeneration and death of retinal pigmented epithelium (RPE), a pigmented and highly specialised cell monolayer which normally performs a number of essential roles in the macula such as phagocytosis of photoreceptor outer segments, secretion of growth factors, transport of nutrients and waste products between the outer retina and the choroid, and secretion of cytokines [2,3]. RPE cells are located between the photoreceptors and the underlying Bruch's membrane (BM), which separates the RPE from the blood vessels of the choroid [2,3]. Even though AMD is considered a priority eye disease for the World Health Organization (WHO) [4], effective therapeutic approaches are still scarce. In this context, cell therapies are considered the most promising solution for the prevention of vision loss, and pluripotent stem cells (PSCs) appear as a unique, unlimited source of specialised RPE cells suitable for transplantation [5]. Indeed, the potential of both human embryonic stem cells (hESCs) and human-induced pluripotent stem cells (hiPSCs) to differentiate into functional RPE cells has been well enough demonstrated before [58].

RPE cells are anchorage-dependent polarised cells with specific apical/basal features $[9,10]$. Using a supportive scaffold can thus ensure that cells adhere and acquire the right orientation to form the interface with the photoreceptors following transplantation. Moreover, because in AMD the underlying $\mathrm{BM}$ is often compromised, the thin scaffold can further act as a prosthetic BM, ensuring the survival, integrity and functionality of the attached RPE cell monolayer [6,11].

In recent years, biomimetic materials that imitate the properties of native extracellular matrices (ECM) have received increasing attention due to their capacity to modulate cell behaviour [9,12]. Even so, few studies so far have attempted to mimic the properties of the BM for RPE cell culture and transplantation [11,13-15]. The BM is a 2-4.7 $\mu \mathrm{m}$ thick semi-permeable membrane consisting of five protein-rich layers: the basement membrane of the RPE (collagen type IV, laminin, fibronectin), the inner collagenous layer (collagens type I, III and V), the elastin layer (elastin, collagen type VI, fibronectin), 
the outer collagenous layer (similar to the inner collagenous layer) and the basement membrane of the choriocapillaris (laminin and collagens type IV, V and VI) $[13,16]$.

One simple method to produce semi-permeable thin films is the breath figure (BF) or water templating method (Figure 1 A) [17]. In this solvent casting method, the polymer is typically dissolved in a volatile, water-immiscible organic solvent, and the solution cast under high relative humidity (RH). Water droplets condense and grow on the cold surface of the evaporating polymer solution and arrange into an ordered hexagonal lattice in order to reduce the free energy. Finally, a porous honeycomb-like pattern is formed after complete evaporation of both the organic solvent and the water droplets [18-20]. The BF method presents clear advantages compared to other methods such as lithography and micro-contact printing - it is inexpensive and fast, it does not require any specific and costly equipment, and it allows a precise control of pore size through control of process parameters e.g. air humidity and polymer concentration [18-20]. In addition, film thickness can be easily modified by changing experimental parameters [19]. Pores and rims of the honeycomb structure can also mimic important microtopographic features of the underlying layers of the native ECM, and the micropatterned surface may even have a positive effect on the properties of cultured RPE cells such as the preservation of their cuboidal morphology [21]. In turn, the presence of pores throughout the scaffold can enable the free flow of nutrients, oxygen and waste products, which is extremely important for cell survival and function in the cell culture medium and in case of transplantation [17].

Recently, we showed that porous honeycomb films dip-coated with collagen type IV enabled the free flow of ions and molecules across the material, and constituted promising materials as cell culture substrates for hESC-RPE cells [17]. In tissue engineering, however, it is widely acknowledged that the ECM and its three-dimensional organisation is important for cell adhesion and cellular response [12,22], and creating a biomimetic microenvironment for RPE cells may be essential for the maintenance of cell survival and function in vivo. In this scenario, the concept of coating the surface of microporous films with organised layers of the native $\mathrm{BM}$ is particularly attractive as a means to create a biomimetic membrane for subretinal cell therapy.

The Langmuir-Blodgett (LB) transfer has shown to be exceptional in the production of biomimetic models with well-controlled molecular organisation, such as collagen substrates [23,24]. The principle 
is that collagen at low $\mathrm{pH}$ occurs in its molecular form, but forms fibres when applied to the interface between air and phosphate buffered saline solution, since the charge neutralisation triggers the association between molecules. When this interface is compressed at a Langmuir trough, collagen fibres become oriented and can subsequently be transferred onto a solid substrate by vertical motion of the substrate into the buffer solution and through the monolayer (Figure 1 B) $[23,25,26]$. Alternatively, highly oriented collagen substrates prepared by horizontal immersion of solid substrates, or LangmuirSchafer (LS) deposition (LS) [27], have been prepared with positive effects over the adhesion and proliferation of 3T3 fibroblasts [25]. Recently, our team has also showed the potential of collagen films prepared by LS deposition onto commercial polymer substrates for RPE cell culture [14]. Even so, the use of LS films as biomaterials for tissue engineering applications is still in its infancy, and, to our knowledge, no records can be found on LS film deposition onto biodegradable polymer surfaces, as inorganic substrates such as mica, silicon and glass are the most commonly elected materials [25,28,29]. In this work, we focused on the main properties of the BM to create a prosthetic BM having properties comparable to that of native membrane. In particular, we applied the BF method to produce semipermeable, porous, biodegradable films, and we took advantage of the LB technique to apply a thin double layer based on human collagen I and human collagen IV on the surface of the porous films. The suitability of the characterised biomaterials as substrates for hESC-RPE was investigated in vitro and the results were compared with the dip-coated controls, prepared by simple adsorption of the two collagen types, and with commercial cell culture inserts.

\section{Materials and methods}

\subsection{Preparation of honeycomb films}

Porous films were prepared by the BF method, generally as described elsewhere [30] and as simply illustrated in Figure 1 A. Solutions of the copolymer 96/4 L-lactide/D-lactide (PLDLA, purified, medical grade, IV midpoint 2.0 dl/g, PURASORB PLD 9620, Corbion Purac, Netherlands) and the surfactant dioleoyl phosphatidylethanolamine (DOPE, Sigma, Japan) were initially prepared in chloroform to a concentration of $10 \mathrm{mg} \mathrm{ml}^{-1}$ and $0.1 \mathrm{mg} \mathrm{ml}^{-1}$, respectively. Aliquots $(500 \mu \mathrm{l})$ of the prepared solutions were cast onto petri dishes (Ø $40 \mathrm{~mm}$, Steriplan®; Figure $1 \mathrm{~A}$-i.) before the solvent was let to evaporate under airflow at $80 \pm 3 \%$ relative humidity (Figure $1 \mathrm{~A}$-ii.). Porous films were then 
air-dried at room temperature (RT) and were subsequently washed three times with $70 \%$ ethanol. Finally, samples were dried again in ambient conditions, and were later kept in a vacuum chamber until further use.

\subsection{Langmuir-Schaefer deposition of collagen onto honeycomb films}

Treatment of human collagen type I and human collagen type IV from human placenta (Sigma-Aldrich St. Louis, MO, USA) essentially followed a previously published protocol [23]. For both collagens, dissolution was carried out in dilute acetic acid (Merck, Germany, $\mathrm{pH} \sim 3$ ) to a concentration of $1 \mathrm{mg}$ $\mathrm{ml}^{-1}$, and solutions were subsequently sonicated in an icy water bath for 10 minutes, followed by a 10minute rest period and by an additional 10-minute sonication period.

The Langmuir-Schaefer (LS) films were prepared using the KSV minitrough system as described by Sorkio et al. [14]. A simplified version of the procedure is shown in Figure 1 B. The subphase consisted of two-fold Dulbecco's phosphate buffered saline (DPBS) pH 7.4 (Lonza Group Ltd., Basel, Switzerland), maintained at $20.8 \pm 0.5^{\circ} \mathrm{C}$. A volume of $180 \mu 1$ of the freshly prepared collagen solution was added drop-wise onto the subphase using a glass microsyringe (Figure 1 B-i.) and the spread collagen film was left to stabilise for 30 minutes. Compression (Figure $1 \mathrm{~B}$-ii.) took place at a speed of $65 \mathrm{~mm} \mathrm{~min}^{-1}$ or $48.75 \mathrm{~cm}^{2} \mathrm{~min}^{-1}$. Films were compressed to the deposition pressure of $12 \mathrm{mN} \mathrm{m}^{-1}$ and $30 \mathrm{mN} \mathrm{m}^{-1}$ for collagen type I and collagen type IV, respectively. After 15 minutes, the stabilised films were deposited onto the substrates, i.e. the honeycomb films and polyethylene terephthalate (PET) cell culture inserts ( $5 \mu \mathrm{m}$ pore size, Millipore), by the touch-and-lift method (Figure 1 B-iii.). Samples were allowed to dry in a desiccator for a minimum of 24 hours. Before further analysis or second LS deposition, samples were washed twice with Milli-Q $\mathrm{H}_{2} \mathrm{O}$ and left again to dry. For the double layer collagen LS films, a type IV collagen film was deposited on top of a dried type I collagen film as described above (i.e. essentially repeating B. in Figure 1 using collagen type IV). Commercial PET inserts, $5 \mu \mathrm{m}$ pore size, were used as control materials.

\subsection{Dip-coating of honeycomb films}

Control samples were prepared by dipping the honeycomb films in collagen I/IV solution. Samples were firstly disinfected with 70\% ethanol and washed with sterile DPBS (2x). Honeycomb films were 
then immersed in a protein DPBS solution ( $\mathrm{pH}$ 7) containing $5 \mu \mathrm{g} \mathrm{cm}^{-2}$ collagen I and $5 \mu \mathrm{g} \mathrm{cm}^{-2}$ collagen IV, for 3 hours at $37^{\circ} \mathrm{C}$. Finally, dip-coated controls were rinsed twice with DPBS (RT) to remove the unbound protein. Dip-coated PET inserts were additionally prepared for comparison.

\subsection{Atomic force microscopy}

Atomic force microscopy (AFM, XE-100, Park Systems Corp, USA) was carried out to analyse the topography of sample surface. Samples were scanned in noncontact mode, under air and at RT using an APPNANO AFM cantilever (type ACTA, $L=125 \mu \mathrm{m}$, tip radius $<10 \mathrm{~nm}, f=200-400 \mathrm{kHz}$, spring constant $=25-75 \mathrm{~N} \mathrm{~m}^{-1}$, coating aluminium). Data were analysed using Park Systems image analysis software (XEI, Park Systems, USA). The arithmetic mean of surface roughness $\left(\mathrm{R}_{\mathrm{a}}\right)$ was determined from a minimum of three randomly chosen $10 \times 10 \mu \mathrm{m}^{2}$ imaged areas using XEI software.

\subsection{Water contact angle}

The hydrophilicity of sample surface was probed by measuring static water contact angles. The sessile drop method was used at RT using a Theta Lite optical tensiometer (Attension, Biolin Scientific AB, Sweden).

\subsection{Immunostaining of collagen type I and IV}

Immunofluorescence studies were carried out to identify the presence of collagen type I and IV on the surface of the dip-coated and LS films. First of all, films were incubated (1h, RT) with $3 \%$ bovine serum albumin (BSA, Sigma-Aldrich, USA), and subsequently with the primary antibodies, i.e. mouse anticollagen IV (Millipore, USA) and rabbit anti-collagen I (Millipore, USA), at a 1:200 ratio in 0.5\% BSADPBS (overnight, $4{ }^{\circ} \mathrm{C}$ ). After washing with DPBS (3x), films were incubated (1h, RT) with the secondary antibodies: donkey anti-mouse Alexa Fluor 488 (Molecular Probes, Life Technologies, USA) and goat anti-rabbit Alexa Fluor 568, both diluted to ratio of 1:800 in 0.5\% BSA in DPBS. Before imaging, films were washed again with DPBS $(3 \mathrm{x})$. Secondary antibody controls were similarly prepared but excluding the incubation step with the primary controls. Collagen distribution on sample surface was imaged using a Zeiss LSM780 Laser Scanning Confocal Microscope (Carl Zeiss, Germany) and a Plan-Apochromat 63x/1.2 (water) objective (Carl Zeiss, Germany). Samples were further compared to the uncoated controls. 


\subsection{Electrical resistance of films}

Electrical resistance $(\mathrm{R})$ studies were carried out to investigate the diffusivity across the honeycomb films [17]. Samples were initially soaked in $70 \%$ ethanol for 5 minutes and then in DPBS for at least 10 minutes. Honeycomb films and controls were mounted into P2307 sliders (Physiologic Instruments, USA) and tighly assembled to a custom-built Teflon chamber. Sliders were submerged in DPBS, and $\mathrm{R}$ values across the materials were monitored using a Millicell electrical resistance system volt-ohm meter (Merck Millipore, Germany). The maximum $\mathrm{R}$ value was established by measuring $\mathrm{R}$ across empty (unloaded) sliders.

2.8. Hydrolytic stability of films and scanning electron microscopy

The hydrolytic stability of the films was investigated by immersing samples $(\sim 5 \mathrm{x} 5 \mathrm{~mm})$ in $1 \mathrm{ml}$ DPBS solution followed by incubation at $37{ }^{\circ} \mathrm{C}$ for 2 and 4 weeks. DPBS solution was replaced weekly. At the end of each timepoint, samples were gently washed with deionised water, dried at RT and their surface analysed by field emission scanning electron microscope (FE-SEM, Carl Zeiss Ultra 55, Germany). Samples used for cell culture (incubated for 8 weeks) were similarly analysed following cell fixation. For clarity, both cell-free and cell-enriched scaffold sections were imaged. After cell culture, samples were washed with DPBS and the materials were fixed in $2.5 \%$ glutaraldehyde solution for $1 \mathrm{~h}$, RT. Thereafter, samples were thoroughly washed with distilled water for $15 \mathrm{~min}$, and were dehydrated in a series of ethanol solutions $(10 \%, 20 \%, 40 \%, 60 \%, 80 \%, 96 \%$ ethanol). Finally, samples were airdried and stored under vacuum until FE-SEM was carried out. Aperture size was always $20 \mu \mathrm{m}$ and the acceleration voltages varied from $1 \mathrm{kV}$ to $3 \mathrm{kV}$ depending on the charging of the sample. Images were taken with and without Au coating. Au was deposited by vacuum thermal evaporation (BOCEdwards Auto-306).

\subsection{Human embryonic stem cell-derived retinal pigment epithelium}

Derivation and characterisation of hESC lines Regea 08/017 (46, XX) and Regea 11/013 (46, XY) were carried out using serum-free conditions as previously described [31]. Culture of the hESCs was performed on top of mitomycin $(10 \mu \mathrm{g} / \mathrm{mL} ; \quad$ SigmaAldrich Corp., St. Louis, MO, USA) treated human foreskin fibroblast feeder cells (CRL2429TM; ATCC, Manassas, VA, USA) as described before [14]. The hESCs were spontaneously 
differentiated into RPE cells in floating cell aggregates followed by enrichment on collagen IV-coated (human placenta, $5 \mu \mathrm{g} \mathrm{cm}^{-2}$; Sigma-Aldrich, MO, USA) well plates in RPE basic medium as previously described [8]. After enrichment, the differentiated RPE cells were seeded on the LS- or freshly dip-coated films and PET cell culture inserts at a density of $1.8 \times 10^{5}$ cells $\mathrm{cm}^{-2}$. Cells were cultured on the materials for a period of 8 weeks in RPE basic medium which was changed three times a week.

\subsection{Immunofluorescence/protein staining}

After cell culture, cells were thoroughly washed with DPBS, fixed with 4\% paraformaldehyde (PFA, 10 min, RT) and washed again three times with DPBS. Cells were subsequently permeabilised with $0.1 \%$ Triton X-100 in DPBS for $10 \mathrm{~min}$ at RT, and were further incubated with 3\% BSA for $1 \mathrm{~h}, \mathrm{RT}$. Appropriate dilutions of the primary antibodies (in 0.5\% BSA-PBS) were added to the samples, and were left to incubate overnight at $4{ }^{\circ} \mathrm{C}$, specifically: mouse anti- $\mathrm{Na}^{+}-\mathrm{K}^{+}$-ATPase (1:200, Abcam, UK), mouse anti-cellular retinaldehyde-binding protein (CRALBP, 1:500, Abcam, UK), rabbit anti-zonula occludens 1 (ZO-1, 1:200, Invitrogen, USA), rabbit anti Claudin-3 (1:80, Thermo Fisher Scientific, USA), and rabbit anti-retinal pigment epithelium-specific $65 \mathrm{kDa}$ protein (RPE65, 1:600, a kind gift from Prof. Michael Redmond, NEI/NIH, USA). After three times washing with DPBS, cells were then incubated with the secondary antibodies i.e. goat anti-rabbit Alexa Fluor 568 (1:800, Molecular Probes, UK) and donkey anti-mouse Alexa Fluor 488 (1:800, Molecular Probes, UK).

Secondary antibody controls were similarly prepared but excluding the incubation step with the primary controls. Cells were finally washed with DPBS and mounted using Vectashield mounting medium (Vector Laboratories, USA) containing 4', 6' diamidino-2-phenylidole (DAPI) for visualisation of the nuclei.

Filamentous actin was visualised following cell fixation and permeabilisation, by incubating samples (20-30 min, RT) with phalloidin-tetramethylrhodamine B isothiocyanate (TRITC, Sigma-Aldrich, USA) prepared in 3\% BSA in DPBS. Cells were washed with DPBS and mounted using Vactashield mounting medium containing DAPI. 
Images were acquired using a Zeiss LSM 780 Confocal Microscope (Carl Zeiss, Germany) or an Olympus BX60 microscope (Olympus, Japan).

2.11. Phagocytosis of photoreceptor outer segments

Phagocytosis of photoreceptor outer segments (POS) by hESC-RPE was investigated after 8 weeks of culture. POS were isolated from porcine eyes, collected to cold AMES' medium (Sigma- Aldrich) with $0.5 \%$ Penicillin-Streptomycin immediately after slaughter and kept on ice, protected from light until POS extraction approximately $2 \mathrm{~h}$ later. The retinas were removed under red light and homogenized with gentle shaking in $0.73 \mathrm{M}$ sucrose phosphate buffer, filtered twice through a gauze and separated in sucrose gradient $(0.75 \mathrm{M}, 1.0 \mathrm{M}, 1.25 \mathrm{M}, 1.5 \mathrm{M}, 1.75 \mathrm{M})$ by ultracentrifugation (Optima ultracentrifuge, Beckman Coulter, Inc., Brea, CA) 112,400g for 48 min at $4^{\circ} \mathrm{C}$. The faint pink POS layer was collected in phosphate buffer, washed twice with centrifugation $3000 \mathrm{~g}$ for $10 \mathrm{~min}$ at $4^{\circ} \mathrm{C}$ and stored as aliquots in $73 \mathrm{mM}$ sucrose phosphate buffer at $-70^{\circ} \mathrm{C}$. For phagocytosis assay, POS were defrosted, centrifuged 3000g for 4 min, washed with DPBS and suspended into RPE basic medium containing 10\% FBS and 5 $\mu \mathrm{g} / \mathrm{ml}$ Plasmocin Prophylactic (Invivogen, France). POS were added to the apical side of the hESC-RPE and incubated protected from light for $4 \mathrm{~h}$ at $37^{\circ} \mathrm{C}$. After incubation, cells were fixed and immunostained as described above. Cells were then labelled with 1:200 dilution of mouse antiopsin antibody (Sigma-Aldrich, USA) overnight at $4^{\circ} \mathrm{C}$. After DPBS washing, cells were labelled with donkey anti-mouse Alexa Fluor 488 (1:400) and Phalloidin-TRITC (1:500) for 1h at RT. Cells were washed again several times with DPBS before being mounted with ProLong Gold antifade reagent containing DAPI (Thermo Fisher Scientific, USA). Z-stack images were acquired with the confocal microscope to visualise internalised POS (LSM 700, Carl Zeiss, 63× oil immersion objective).

\subsection{Statistical analysis}

All numerical data are shown as mean and standard deviation. Statistical analysis was performed by one-way analysis of variance (ANOVA) and the post-hoc Tukey or Games-Howell multiple comparisons tests.

\section{Results}

3.1. Physical properties of the porous films 
PLDLA films prepared in this study were approximately $20 \mu \mathrm{m}$ thick and were characterised by a honeycomb-like surface with average pore diameter of $5 \mu \mathrm{m}$, as shown by the AFM micrograph in Figure $2 \mathrm{~A}$ and respective height topographical profile. This highly regular surface structure was not affected by dip-coating the samples with collagens type I and IV (Figure 2 B). Even so, an effective decrease of surface roughness $R_{a}$ was observed (Figure 3 A) following the physical adsorption of collagen to the surface. Interestingly, the LS deposition of the bilayer collagen type I/collagen type IV onto the honeycomb substrate resulted in an evident coverage of the pores (Figure $2 \mathrm{C}$ ), with the concomitant reduction of surface roughness to the nanometer scale, as shown by the height topographical profile (Figure $2 \mathrm{C}$ ) and by the $\mathrm{R}_{\mathrm{a}}$ values in Figure $3 \mathrm{~A}$. AFM images of the commercial PET inserts (5 $\mu \mathrm{m}$ pore size) used as controls show a lower porosity and heterogeneous distribution of pores as compared to the honeycomb films (Figure 2 D). Furthermore, uncoated PET inserts were not significantly different from the dip-coated surfaces (Figure $2 \mathrm{E}$ ) or from surfaces that underwent collagen deposition by the LS method (Figure $2 \mathrm{~F}$ ), which was additionally confirmed by the $\mathrm{R}_{\mathrm{a}}$ values (Figure $3 \mathrm{~A}$; differences not significant at the 0.05 level).

The hydrophilicity of the membranes was assessed by measuring the water contact angle (WCA) of the surfaces (Figure 3 B). PLDLA honeycomb films were shown to have WCA values of approximately $125^{\circ}$, in consistency with the hydrophobic nature of the polymer and porous nature of the membranes, but the values were found to decrease when the surfaces were coated with collagen and thereby became more hydrophilic. Expressively, reduction of WCA was much more pronounced when the surface was coated with the LS films (average $66.8^{\circ}$ ), as compared to the dip-coated surfaces (average $116^{\circ}$ ). Technical difficulties related with the high hydrophilicity and large pore size of PET controls hindered the accurate measurement of the WCA for these controls which are thus not displayed in Figure $3 \mathrm{~B}$. However, the literature features agreeing values of approximately $75^{\circ}$ to PET membranes [32-34], which will be used as a reference for our studies.

\subsection{Immunofluorescence of collagen type I and IV}

Immunofluorescence studies were carried out to investigate the presence and distribution of collagen type I and IV on the surface of the porous membranes. Collagens were well distributed along the rims of the dip-coated honeycomb membranes and the pores were clearly left uncovered, as shown in Figure 
4 A and respective vertical confocal sections. This strikingly differed from the honeycomb-LS surfaces, where both rims and pores were completely covered by the proteins. In fact, the vertical confocal sections in Figure 4 B demonstrate that the surface was completely and homogeneously covered by the deposited collagen films. The surface of dip-coated control PET membranes showed similar surface coverage to the PET-LS controls, characterised by large, homogeneous coverage of the membranes with nonetheless uncoated pores (Figures $4 \mathrm{C}$ and $\mathrm{D}$, respectively). Notwithstanding this observation, collagen type IV on PET-LS membranes showed a marked presence along the walls of the pores (vertical confocal sections in Figure 4 D). In all cases, collagen type IV was present in higher amounts than collagen I.

\subsection{Electrical resistance}

Electrical resistance through the membranes $(\mathrm{R})$ was also measured as a means to compare the permeability of the samples to small species in solution. The results in Figure 5 show that the adsorption or deposition of collagen did not significantly affect the flow of ions through the honeycomb membranes. In a similar manner, the adsorption of collagen by dip-coating of PET membranes did not cause significant differences in R. However, $\mathrm{R}$ was significantly reduced by the LS deposition of collagen onto PET membranes. In all cases, $\mathrm{R}$ was expressively lower than the reference value of $\mathrm{R}$ for mature hESC-RPE [8].

\subsection{Mechanical testing and hydrolytic stability of films}

Tensile testing of honeycomb-LS films was carried out to evaluate their mechanical properties and thus their potential in RPE transplantation (Methods shown in Supplementary Material). Samples were tested until they broke and the maximum strengths were recorded. According to the measurements, the tensile strength of the LS-coated honeycomb film was 7.7 $\pm 0.5 \mathrm{MPa}$. Presumably, the LS-coating had no notable influence on the strength of the film.

The in vitro stability of the deposited collagen layers was investigated by SEM (Figure 6) following immersion of the membranes in DPBS for 4 weeks, and at the end of the cell culture ( 8 weeks). Dipcoated honeycomb membranes largely retained the surface structure, with intact surface rims being observed even after 8 weeks. The underlying porous polymer layers, formed as the solvent evaporated in the BF method, are additionally visible in the SEM micrographs. LS-coated honeycomb membranes 
were shown not only to retain the underlying porous structure of PLDLA, but also to retain at least for 4 weeks most of the collagen LS films. After 2 weeks of immersion, only small holes can be observed on the collagen layer, and after 4 weeks some pores are already uncovered, even though the majority of the collagen layer is still present. SEM micrographs did not show any significant differences between uncoated, dip-coated and LS-coated PET controls and after the immersion period (not shown). The surface of these control samples was always smooth and the pores uncovered by the collagen layer.

\subsection{Cell culture}

Cell adhesion, spreading and maturation was assessed with two separate hESC-RPE cell lines by bright field and immunofluorescence microscopy. Both cell lines exhibited similar results. Representative images of cell line 08/017 are shown in Figure 7 and supplementary Figures S1 and S2. Maturation status of the cells was evaluated by the expression and subcellular localisation of an apical polarisation marker $\mathrm{Na}^{+}-\mathrm{K}^{+}$-ATPase, visual cycle proteins CRALBP and RPE65, and tight junction proteins ZO-1 and Claudin-3. Low magnification images (bright field and F-actin) show that hESC-RPE adhered and spread on dip-coated honeycomb films, even though aggregates were sometimes observed. In areas where the epithelium was confluent, cells were also significantly pigmented. Cells cultured on dipcoated honeycomb films also demonstrated the typical cobblestone morphology of mature RPE cells (solid arrow, upper image in third panel) as well as elongated morphology (dashed arrow, upper image in third panel). $\mathrm{Na}^{+}-\mathrm{K}^{+}$-ATPase was widely present in the epithelium and, depending on the area, located either on the apical membrane or still on the lateral membrane, as shown in the vertical confocal sections of Figure 7. Even so, CRALBP (vertical confocal sections in Figure 7, last vertical panel) and RPE65 (Supplementary Figure S1) were expressed after 8 weeks of cell culture. ZO-1 was observed in the cell junctions (vertical confocal sections in Figure 7, last vertical panel), but Claudin-3 showed some diffuse staining in addition to localisation to lateral cell membranes (Supplementary Figure S2).

Significantly better cell adherence and spreading was observed when cells were cultured on the honeycomb films covered with the LS collagen layers, with hESC-RPE forming a confluent and uniform pigmented epithelium (low magnification images in Figure 7). Furthermore, honeycomb-LS films (as well as the dip-coated controls) effectively restrained cell migration to the opposite side of the scaffold, as shown by SEM imaging of the cultured surface and of the opposite film surface 
(Supplementary Figure S3). Filamentous actin was nicely organised and low in stress fibres. Cells were shown to have the typical cobblestone morphology of mature RPE cells and expressed $\mathrm{Na}^{+}-\mathrm{K}^{+}$-ATPase on the apical membrane in consistency with a mature and polarised epithelium. Furthermore, cells were positive for the presence of ZO-1 and Claudin-3 in the tight junctions, and expressed CRALBP and RPE65 (Figure 7 and Supplementary Figures S1 and S2).

Both dip-coated PET and PET-LS controls enabled cell adherence and proliferation. However, cells cultured on dip-coated controls often showed elongated or uneven morphology (examples indicated by the dashed arrows on the image showing the $\mathrm{Na}^{+}-\mathrm{K}^{+}$-ATPase marker, third vertical panel) together with the typical cobblestone morphology (example indicated by the solid arrow). Furthermore, actin filaments formed bundles of stress fibres. $\mathrm{Na}^{+}-\mathrm{K}^{+}$-ATPase was poorly expressed in some areas and found on the basal, lateral and apical membranes. In contrast, PET-LS controls showed good cell morphology, a well-organised actin network, and better expression of $\mathrm{Na}^{+}-\mathrm{K}^{+}$-ATPase which was nonetheless not fully polarised to the apical surface (vertical confocal section). Both controls showed some expression of CRALBP and ZO-1, but in many parts, ZO-1 was not fully localised at the tight junctions.

\subsection{Phagocytosis of outer segments}

The functionality of hESC-RPE cells on honeycomb films and PET inserts after collagen dip-coating or LS deposition was assessed by an in vitro phagocytosis assay using isolated porcine POS after 8 weeks of culture using two hESC-RPE cell lines. Internalised POS were seen in cells on all examined substrates (Figure 8).

\section{Discussion}

The manufacture of an artificial BM and transplantation of RPE cells is currently seen as the most promising solution for the treatment of degenerative eye diseases such as AMD. In our work, we focused on the properties of the BM to develop a biomaterial with resemblance to the native ECM layers, capable of providing the best microenvironment for tissue regeneration. The prepared materials were semi-permeable like the BM, and contained collagen type IV, the main component of the basement membrane of RPE, as well as collagen type I, which is abundant in the inner collagenous layer $[13,35]$. In addition, collagen fibres in the inner collagenous layer are naturally disposed in a nanometre-thick 
multilayered structure parallel to the plane of the BM [35], and the LS deposition method is ideal to mimic such structures $[14,25]$.

Here, $20 \mu \mathrm{m}$-thick films were prepared as support material to the LS collagen layers, having in mind the need to easily manipulate the biomaterial for in vitro cell culture and potential surgical implantation. PLDLA was used as the scaffold-forming material, due to the widely recognised biocompatibility and biomimetic biodegradability of poly(lactic acid) substrates [36]. In addition, our previous studies [17] showed that highly permeable PLDLA porous films could be prepared by careful selection of the solvent casting conditions, particularly using high humidity $(80 \% \mathrm{RH})$, and an adequate polymer concentration and surfactant to polymer ratio. This careful selection allows us to control pore size to approximately 5 $\mu \mathrm{m}$, as shown in Figure $2 \mathrm{~A}$, which is large enough to enable the flow of small molecules and ions, but small enough to prevent the migration of cultured or mature RPE cells across the material [17].

Another important feature of the poly(lactic acid) substrates is their hydrophobicity, which is generally known to favour protein adsorption $[37,38]$. As such, it was anticipated that the surface of the PLDLA substrates would favour the adsorption/deposition of the two ECM proteins, as observed in our previous studies on collagen IV adsorption onto honeycomb films [17]. Indeed, the decreased $\mathrm{R}_{\mathrm{a}}$ values (Figure 3 A) together with the reduction of WCA (Figure 3 B) following the dip-coating procedure with the two collagens (controls) immediately suggests the effective adsorption of the proteins onto the material surface, which thereby became more hydrophilic and smooth. Notwithstanding this fact, it is expected that collagen was physically adsorbed in a more or less random way to the rims of the honeycomb structure as observed before for dip-coated controls [14], leaving the pores clearly uncovered, as confirmed by the AFM micrographs in Figure 2 and the immunofluorescence images in Figure 4 A.

Significantly more striking were the results for the deposition of the two consecutive layers of collagen by the LS technique, which not only caused the surface to become expressively more hydrophilic and smooth (Figure 3), but also the pores to become completely covered by the ECM proteins (Figure 2 and Figure 4 B). Collagen molecules, consisting mainly of hydrophobic domains, spread nicely on the airwater interface and form stable films upon compression [26]. Thereafter, collagen molecules bind to the substrate via hydrophobic-hydrophobic interactions with fibres being aligned parallel to the Langmuir trough barriers (or perpendicular to the direction of the applied compression) $[14,25,26]$. In 
this context, it is likely that the high deposition of the ECM proteins is aided by the hydrophobic nature of the PLDLA substrate. Furthermore, two factors can account for the substantial increase in the hydrophilicity of the substrate: firstly, the interactions between the hydrophobic groups of the substrate and collagen entail the exposure of the hydrophilic domains on the surface; secondly, according to the Wenzel's and Cassie's theories, hydrophobic surfaces become more hydrophobic when the surface is rough [39], and consequently when the pores of the honeycomb films get covered, both surface $R_{a}$ and hydrophobicity decrease.

In this work, commercial PET inserts $(5 \mu \mathrm{m}$ pore size $)$ were used as control materials due to their common use in cell culture $[14,40]$, and were thus similarly characterised and tested as substrates for hESC-RPE. The fact that surface roughness did not change significantly with the adsorption or deposition of collagen types I and IV (Figures 2 and 3 A) is consistent with previous results showing that the thickness of the double layer LS films was only of a few tens of nanometres and even lower for dip-coated controls [14], and with the fact that the pores were found to be largely uncovered by the ECM proteins. In turn, the reason for the low pore coverage in PET inserts is unclear, but possibly related with the increased hydrophilicity of the substrate - and thus lower tendency for the establishment of hydrophobic interactions between collagen and the solid surface - as compared to the hydrophobic PLDLA substrates.

One important observation from a qualitative point of view concerns the distribution of collagen type I and collagen type IV on the surface of the porous films. In all cases it was patent that collagen type IV - the topmost LS layer - was more widely distributed than the type I counterpart. For the LS films, this observation has been related with the higher amount of collagen IV present at the air-subphase interface after collagen spreading and fibre formation. In fact, it is observed that for the same mass/area of collagen spread at the air-water interface, collagen type IV can be compressed to much higher surface pressures and thus smaller areas than collagen type I, originating thicker films [14]. However, the fact that collagen type IV was also present in higher amounts for the dip-coated controls suggests that the differences may be primarily related with the supramolecular nature of the protein itself, as type I collagen is known to form fibres, and type IV a two-dimensional reticulum [41]. 
In our previous research, the apparent permeability coefficient $\mathrm{P}_{\text {app }}$ for similarly prepared uncoated honeycomb films was in the order $\approx 2 \times 10^{-4} \pm 8 \times 10^{-5}$ due to the large porosity of the scaffolds; the permeability was also shown to inversely correlate with $\mathrm{R}$, i.e. higher permeability values were associated with lower R [17]. In spite of its poor use so far as a characterisation tool for honeycomb films, the method is fast, simple and inexpensive, and it provides a good estimation of the diffusion capacity of porous membranes $[17,42]$. In this work, the fact that $\mathrm{R}$ was mostly not affected by the adsorption/LS deposition of collagen onto the porous films (with the exception of the PET-LS control) is in consistency with the increased hydrophilicity and wettability of the collagen-coated films. Furthermore, double layer collagen LS films have been shown to be only a few tens of nanometres thick [14], thereby not affecting the permeability of the honeycomb films, even if the pores on the surface are covered by the ECM proteins. These results suggest that the diffusion of oxygen, nutrients and waste products between the choroid blood vessels and the outer retina will be maintained in vivo. Importantly, the value of $\mathrm{R}$ for all samples and controls was at least 7 times lower than the reported values for mature hESC-RPE epithelia [8], which means that the pigmented epithelium will be the rate-limiting factor to the diffusion of small species and not the biomaterial itself.

While the manufactured materials demonstrated the presence of the two collagen types as well as the maintenance of the diffusion properties by the highly porous structure, the material must also prove to be stable enough to be handled surgically and to withstand the in vitro and in vivo environments. Preliminary mechanical testing on honeycomb-LS films confirmed tensile strength values $(7.7 \pm 0.5$ MPa) that were in the same order as those previously reported for similar materials, such as other porous PLA membranes (1 to $8 \mathrm{MPa}$, [43]; 8.3 MPa [44]). Only a small number of studies indicate the tensile strength of thin materials that were surgically implanted. In one case, the significantly lower tensile strength value ( $0.3 \mathrm{MPa})$ reported for collagen hydrogel implants (regeneration of the cornea) motivated the use of overlying sutures [45]. Despite the low mechanical properties, the collagen implants showed sufficient characteristics for transplantation. Cross-linked collagen hydrogel sheets $(200-230 \mu \mathrm{m})$ grafted into the cornea of mice that showed to be mechanically resistant to handling and suturing showed tensile strengths of only about $2 \mathrm{MPa}$ [46]. According to these findings and to our own 
experience in handling the films, it is expected that the LS-coated honeycomb films can be surgically implanted without effort.

Another aspect that is often neglected when developing new materials for RPE transplantation is the hydrolytic susceptibility of the material in vitro or during the regeneration of the damaged tissue. Here, we confirmed that the honeycomb structure is completely retained for at least 4 weeks following immersion in DPBS and for the 8 weeks of cell culture, whereas the LS-deposited collagen layers are mostly preserved for at least 4 weeks. In vivo, RPE cells have been shown to produce the ECM components of the BM, namely collagen types I, II, III and IV, in addition to laminin and fibronectin, whereas in vitro this can already be observed after 3 days in culture [47]. In this context, it is expected that the production of ECM proteins will happen in vitro soon after RPE cell adhesion, and will continue in vivo after transplantation of the prosthetic BM. The slow degradation of the biomaterial should thus provide enough time for the complete integration of the biomaterial with the surrounding retinal tissue. Importantly, from a toxicological point of view, the slow degradation of the material, together with the observed diffusion capacity of the membranes, will expectedly contribute to maintain the degradation products of the polymer to a minimum at the implantation site.

Mature RPE cells are pigmented cells typically characterised by a hexagonal cobblestone-like morphology that form a tightly packed epithelium $[8,48]$. Right from the observation of these simple features, it is already apparent that the layered and highly organised nanostructure of the LS films (both for the honeycomb film and PET control) favours the preservation of the RPE phenotype, as well as the adhesion and spreading of cells, as compared to the substrates where collagen is randomly adsorbed to the samples. In fact, the occasional presence of elongated fibroblast-like cells on the dip-coated controls is reminiscent of cell dedifferentiation and loss of specific cell functions [49].

Actin filaments are an important component of the cytoskeleton and a good indicator of the forces generated with the substrate and between cells [21,50]. With the exception of the dip-coated PET insert, all samples showed the presence of short actin filaments arranged into circumferential rings, which is suggestive of a mature and tight epithelium where cell-cell interactions dominate. In contrast, the dipcoated PET control showed abundant presence of stress fibres; these bundles of actin filaments are 
typically anchored to focal adhesions necessary for cell-ECM interactions during adhesion and migration [50], and their presence suggests an early stage of maturation [51].

Studies have shown that the formation of tight junctions and the expression of ZO-1 and Claudin proteins is essential to establish the blood-retina barrier [52-54]. The fact that both ZO-1 (Figure 7) and Claudin-3 (Supplementary Figure S2) were detected at the tight junctions of hESC-RPE cultured on LS-coated substrates, and especially on the honeycomb-LS film, confirms that the epithelia developed the retinal barrier function. In contrast, the fact that Claudin-3 was not fully localised at the tight junctions of cells cultured on the PET controls indicates poor barrier properties or incomplete maturation for these controls. The establishment of tight junctions between RPE cells is additionally essential to establish the apical to basolateral polarity [10]. $\mathrm{Na}^{+}-\mathrm{K}^{+}$-ATPase is a membrane protein essential in transepithelial transport that is expressed and becomes apically polarised in the early stage of tight junction development [10]. The superior expression and apical localisation of $\mathrm{Na}^{+}-\mathrm{K}^{+}-\mathrm{ATPase}$ in the honeycomb-LS compared to the controls confirms that the micro and nanostructure of the substrate is essential for tight junction development and polarisation of hESC-RPE cells. Our studies also showed the detection of the CRALBP and RPE65 markers in all samples, proteins with central roles in the visual cycle [55,56], which are typically up-regulated in mature RPE cells.

As mentioned previously, one of the many roles of RPE cells is to perform phagocytosis of the photoreceptor outer segments (POS). Specifically, the photoreceptors constantly undergo a renewal process where the tips of the photoreceptors containing a number of radical species, photo-damaged proteins and lipids are eliminated by the clearing action of RPE [10,57]. In this context, the assessment of the phagocytic capacity of RPE towards POS can be a valuable tool to evaluate the functionality of RPE cells cultured in vitro $[8,14,58-60]$. In spite of clear morphological differences between epithelia on LS- and dip-coated scaffolds, dip-coated membranes showing more fusiform RPE cells typically associated with less mature phenotype [61], cells with phagocytic activity could be detected on all materials, regardless of the coating type.

\section{Conclusions}

The combination of BF and LS technologies enabled the production of biomimetic, biodegradable biomaterials for the culture of hESC-RPE. The resulting materials were porous and semi-permeable but 
had a smooth and hydrophilic surface due to the LS deposition of the collagen IV/collagen I layers. Comparison with the dip-coated and PET controls demonstrated the importance of the micro and nanostructure of the biomaterial for hESC-RPE adhesion, spreading, morphology, protein expression and function. The promising results shown in this work may pave the way to the development of enhanced materials with a close structural (multilayered) resemblance with the native BM, and to the production of prosthetic basement membranes for RPE replacement therapy in the treatment of degenerative eye diseases such as AMD.

\section{Acknowledgments}

This study was supported by the Finnish funding agency of innovation (Tekes) Human Spare Parts program, the Academy of Finland (grant numbers 304909, 253134, 218050, 272808, 133879), the Finnish Cultural Foundation and Päivikki and Sakari Sohlberg Foundation. Suvi Heinämäki, Outi Melin, Hanna Pekkanen and Outi Heikkilä are thanked for technical assistance.

\section{References}

[1] W.L. Wong, X. Su, X. Li, C.M.G. Cheung, R. Klein, C.-Y. Cheng, T.Y. Wong, Global prevalence of age-related macular degeneration and disease burden projection for 2020 and 2040: a systematic review and meta-analysis, Lancet Glob. Health. 2 (2014) e106e116.

[2] K. Kaarniranta, D. Sinha, J. Blasiak, A. Kauppinen, Z. Veréb, A. Salminen, M.E. Boulton, G. Petrovski, Autophagy and heterophagy dysregulation leads to retinal pigment epithelium dysfunction and development of age-related macular degeneration, Autophagy. 9 (2013) 973-984.

[3] R.D. Jager, W.F. Mieler, J.W. Miller, Age-related macular degeneration, N. Engl. J. Med. 358 (2008) 2606-2617.

[4] WHO | Prevention of Blindness and Visual Impairment | Priority eye diseases, WHO. (2016). http://www.who.int/blindness/causes/priority/en/index7.html (accessed July 17, 2016). 
[5] M. Riera, L. Fontrodona, S. Albert, D.M. Ramirez, A. Seriola, A. Salas, Y. Muñoz, D. Ramos, M.P. Villegas-Perez, M.A. Zapata, A. Raya, J. Ruberte, A. Veiga, J. GarciaArumi, Comparative study of human embryonic stem cells (hESC) and human induced pluripotent stem cells (hiPSC) as a treatment for retinal dystrophies, Mol. Ther. Methods Clin. Dev. 3 (2016) 16010.

[6] H. Skottman, Differentiation of human embryonic stem cells and human induced pluripotent stem cells into retinal pigment epithelium, in: M.A. Hayat (Ed.), Stem Cells Cancer Stem Cells Vol. 7, Springer Netherlands, 2012: pp. 187-194.

[7] T.J. Rowland, D.E. Buchholz, D.O. Clegg, Pluripotent human stem cells for the treatment of retinal disease, J. Cell. Physiol. 227 (2012) 457-466.

[8] H. Vaajasaari, T. Ilmarinen, K. Juuti-Uusitalo, K. Rajala, N. Onnela, S. Narkilahti, R. Suuronen, J. Hyttinen, H. Uusitalo, H. Skottman, Toward the defined and xeno-free differentiation of functional human pluripotent stem cell-derived retinal pigment epithelial cells, Mol. Vis. 17 (2011) 558-575.

[9] A.J. Treharne, M.C. Grossel, A.J. Lotery, H.A. Thomson, The chemistry of retinal transplantation: the influence of polymer scaffold properties on retinal cell adhesion and control, Br. J. Ophthalmol. 95 (2011) 768-773.

[10] O. Strauss, The retinal pigment epithelium in visual function, Physiol. Rev. 85 (2005) $845-881$.

[11] P.H. Warnke, M. Alamein, S. Skabo, S. Stephens, R. Bourke, P. Heiner, Q. Liu, Primordium of an artificial Bruch's membrane made of nanofibers for engineering of retinal pigment epithelium cell monolayers, Acta Biomater. 9 (2013) 9414-9422.

[12] M.B. Rahmany, M. Van Dyke, Biomimetic approaches to modulate cellular adhesion in biomaterials: A review, Acta Biomater. 9 (2013) 5431-5437. 
[13] J.C. Booij, D.C. Baas, J. Beisekeeva, T.G.M.F. Gorgels, A.A.B. Bergen, The dynamic nature of Bruch's membrane, Prog. Retin. Eye Res. 29 (2010) 1-18.

[14] A.E. Sorkio, E.P. Vuorimaa-Laukkanen, H.M. Hakola, H. Liang, T.A. Ujula, J.J. ValleDelgado, M. Österberg, M.L. Yliperttula, H. Skottman, Biomimetic collagen I and IV double layer Langmuir-Schaefer films as microenvironment for human pluripotent stem cell derived retinal pigment epithelial cells, Biomaterials. 51 (2015) 257-269.

[15] A. Komez, E.T. Baran, U. Erdem, N. Hasirci, V. Hasirci, Construction of a patterned hydrogel—fibrous mat bilayer structure to mimic choroid and Bruch's membrane layers of retina, J. Biomed. Mater. Res. A. 104 (2016) 2166-2177.

[16] R.S. Ramrattan, der S. Van, C.M. Mooy, B. De, P.G.H. Mulder, J. De, Morphometric analysis of Bruch's membrane, the choriocapillaris, and the choroid in aging, Invest. Ophthalmol. Vis. Sci. 35 (1994) 2857-2864.

[17] M.T. Calejo, T. Ilmarinen, H. Jongprasitkul, H. Skottman, M. Kellomäki, Honeycomb porous films as permeable scaffold materials for human embryonic stem cell-derived retinal pigment epithelium, J. Biomed. Mater. Res. A. 104 (2016) 1646-1656.

[18] A. Muñoz-Bonilla, M. Fernández-García, J. Rodríguez-Hernández, Towards hierarchically ordered functional porous polymeric surfaces prepared by the breath figures approach, Prog. Polym. Sci. 39 (2014) 510-554.

[19] A. Zhang, H. Bai, L. Li, Breath figure: a nature-inspired preparation method for ordered porous films, Chem. Rev. 39 (2015) 510-554.

[20] M. Du, P. Zhu, X. Yan, Y. Su, W. Song, J. Li, Honeycomb self-assembled peptide scaffolds by the breath figure method, Chem. - Eur. J. 17 (2011) 4238-4245.

[21] L. Lu, L. Kam, M. Hasenbein, K. Nyalakonda, R. Bizios, A. Göpferich, J.F. Young, A.G. Mikos, Retinal pigment epithelial cell function on substrates with chemically micropatterned surfaces, Biomaterials. 20 (1999) 2351-2361. 
[22] J. Barthes, H. Özçelik, M. Hindié, A. Ndreu-Halili, A. Hasan, N.E. Vrana, Cell microenvironment engineering and monitoring for tissue engineering and regenerative medicine: the recent advances, BioMed Res. Int. 2014 (2014).

[23] A.J.J. Goffin, J. Rajadas, G.G. Fuller, Interfacial flow processing of collagen, Langmuir. 26 (2010) 3514-3521.

[24] Q. Chen, S. Xu, R. Li, X. Liang, H. Liu, Network structure of collagen layers absorbed on LB film, J. Colloid Interface Sci. 316 (2007) 1-9.

[25] L. Pastorino, E. Dellacasa, S. Scaglione, M. Giulianelli, F. Sbrana, M. Vassalli, C. Ruggiero, Oriented collagen nanocoatings for tissue engineering, Colloids Surf. B Biointerfaces. 114 (2014) 372-378.

[26] A. Tenboll, B. Darvish, W. Hou, A.-S. Duwez, S.J. Dixon, H.A. Goldberg, B. Grohe, S. Mittler, Controlled deposition of highly oriented type I collagen mimicking in vivo collagen structures, Langmuir ACS J. Surf. Colloids. 26 (2010) 12165-12172.

[27] I. Langmuir, V.J. Schaefer, Activities of urease and pepsin monolayers, J. Am. Chem. Soc. 60 (1938) 1351-1360.

[28] M.A. Biesalski, A. Knaebel, R. Tu, M. Tirrell, Cell adhesion on a polymerized peptideamphiphile monolayer, Biomaterials. 27 (2006) 1259-1269.

[29] M. Sakuma, Y. Kumashiro, M. Nakayama, N. Tanaka, K. Umemura, M. Yamato, T. Okano, Control of cell adhesion and detachment on Langmuir-Schaefer surface composed of dodecyl-terminated thermo-responsive polymers, J. Biomater. Sci. Polym. Ed. 25 (2014) 431-443.

[30] Y. Fukuhira, E. Kitazono, T. Hayashi, H. Kaneko, M. Tanaka, M. Shimomura, Y. Sumi, Biodegradable honeycomb-patterned film composed of poly(lactic acid) and dioleoylphosphatidylethanolamine, Biomaterials. 27 (2006) 1797-1802. 
[31] H. Skottman, Derivation and characterization of three new human embryonic stem cell lines in Finland, Vitro Cell. Dev. Biol. - Anim. 46 (2010) 206-209.

[32] I. Donelli, P. Taddei, P.F. Smet, D. Poelman, V.A. Nierstrasz, G. Freddi, Enzymatic surface modification and functionalization of PET: A water contact angle, FTIR, and fluorescence spectroscopy study, Biotechnol. Bioeng. 103 (2009) 845-856.

[33] Z. Fang, J. Yang, Y. Liu, T. Shao, C. Zhang, Surface treatment of polyethylene terephthalate to improving hydrophilicity using atmospheric pressure plasma jet, IEEE Trans. Plasma Sci. 41 (2013) 1627-1634.

[34] N. Inagaki, K. Narushim, N. Tuchida, K. Miyazaki, Surface characterization of plasmamodified poly(ethylene terephthalate) film surfaces, J. Polym. Sci. Part B Polym. Phys. 42 (2004) 3727-3740.

[35] C.A. Curcio, M. Johnson, Structure, Function, and Pathology of Bruch's Membrane, in: Retina, 5th ed., Elsevier, London, 2013: pp. 465-481.

[36] P.X. Ma, Biomimetic materials for tissue engineering, Adv. Drug Deliv. Rev. 60 (2008) 184-198.

[37] S. Foldberg, M. Petersen, P. Fojan, L. Gurevich, T. Fink, C.P. Pennisi, V. Zachar, Patterned poly(lactic acid) films support growth and spontaneous multilineage gene expression of adipose-derived stem cells, Colloids Surf. B Biointerfaces. 93 (2012) 9299.

[38] A. Gessner, R. Waicz, A. Lieske, B.-R. Paulke, K. Mäder, R.H. Müller, Nanoparticles with decreasing surface hydrophobicities: influence on plasma protein adsorption, Int. J. Pharm. 196 (2000) 245-249.

[39] A. Marmur, Wetting on hydrophobic rough surfaces: To be heterogeneous or not to be?, Langmuir. 19 (2003) 8343-8348. 
[40] C. Chollet, C. Chanseau, M. Remy, A. Guignandon, R. Bareille, C. Labrugère, L. Bordenave, M.-C. Durrieu, The effect of RGD density on osteoblast and endothelial cell behavior on RGD-grafted polyethylene terephthalate surfaces, Biomaterials. 30 (2009) $711-720$.

[41] H. Lodish, A. Berk, S.L. Zipursky, P. Matsudaira, D. Baltimore, J. Darnell, Collagen: the fibrous proteins of the matrix, in: Mol. Cell Biol., 4th ed., W. H. Freeman, New York, 2000. http://www.ncbi.nlm.nih.gov/books/NBK21582/ (accessed August 12, 2016).

[42] A. Carbonaro, R. Walczak, P.M. Calderale, M. Ferrari, Nano-pore silicon membrane characterization by diffusion and electrical resistance, J. Membr. Sci. 241 (2004) 249_ 255.

[43] T. Phaechamud, S. Chitrattha, Pore formation mechanism of porous poly(dl-lactic acid) matrix membrane, Mater. Sci. Eng. C. 61 (2016) 744-752.

[44] X.H. Wang, S. Shi, G. Guo, S.Z. Fu, M. Fan, F. Luo, X. Zhao, Y.Q. Wei, Z.Y. Qian, Preparation and characterization of a porous scaffold based on poly(D,L-lactide) and Nhydroxyapatite by phase separation, J. Biomater. Sci. Polym. Ed. 22 (2011) 1917-1929.

[45] P. Fagerholm, N.S. Lagali, J.A. Ong, K. Merrett, W.B. Jackson, J.W. Polarek, E.J. Suuronen, Y. Liu, I. Brunette, M. Griffith, Stable corneal regeneration four years after implantation of a cell-free recombinant human collagen scaffold, Biomaterials. 35 (2014) $2420-2427$.

[46] J.-I. Ahn, L. Kuffova, K. Merrett, D. Mitra, J.V. Forrester, F. Li, M. Griffith, Crosslinked collagen hydrogels as corneal implants: Effects of sterically bulky vs. non-bulky carbodiimides as crosslinkers, Acta Biomater. 9 (2013) 7796-7805.

[47] P.A. Campochiaro, J.A. Jerdon, B.M. Glaser, The extracellular matrix of human retinal pigment epithelial cells in vivo and its synthesis in vitro., Invest. Ophthalmol. Vis. Sci. 27 (1986) 1615-1621. 
[48] C. Brandl, S.J. Zimmermann, V.M. Milenkovic, S.M.G. Rosendahl, F. Grassmann, A. Milenkovic, U. Hehr, M. Federlin, C.H. Wetzel, H. Helbig, B.H.F. Weber, In-depth characterisation of retinal pigment epithelium (RPE) cells derived from human induced pluripotent stem cells (hiPSC), Neuromolecular Med. 16 (2014) 551-564.

[49] L. Lu, M.J. Yaszemski, A.G. Mikos, Retinal pigment epithelium engineering using synthetic biodegradable polymers, Biomaterials. 22 (2001) 3345-3355.

[50] S. Tojkander, G. Gateva, P. Lappalainen, Actin stress fibers - assembly, dynamics and biological roles, J. Cell Sci. 125 (2012) 1855-1864.

[51] V. Savolainen, K. Juuti-Uusitalo, N. Onnela, H. Vaajasaari, S. Narkilahti, R. Suuronen, H. Skottman, J. Hyttinen, Impedance spectroscopy in monitoring the maturation of stem cell-derived retinal pigment epithelium, Ann. Biomed. Eng. 39 (2011) 3055-3069.

[52] K. Konari, N. Sawada, Y. Zhong, H. Isomura, T. Nakagawa, M. Mori, Development of the blood-retinal barrier in vitro: Formation of tight junctions as revealed by occludin and ZO-1 correlates with the barrier function of chick retinal pigment epithelial cells, Exp. Eye Res. 61 (1995) 99-108.

[53] C. Chang, X. Wang, R.B. Caldwell, Serum opens tight junctions and reduces ZO-1 protein in retinal epithelial cells, J. Neurochem. 69 (1997) 859-867.

[54] L.J. Rizzolo, Barrier properties of cultured retinal pigment epithelium, Exp. Eye Res. 126 (2014) 16-26.

[55] F. Gonzalez-Fernandez, Evolution of the visual cycle: the role of retinoid-binding proteins, J. Endocrinol. 175 (2002) 75-88.

[56] T.M. Redmond, S. Yu, E. Lee, D. Bok, D. Hamasaki, N. Chen, P. Goletz, J.X. Ma, R.K. Crouch, K. Pfeifer, Rpe65 is necessary for production of 11-cis-vitamin A in the retinal visual cycle, Nat. Genet. 20 (1998) 344-351. 
[57] F. Mazzoni, H. Safa, S.C. Finnemann, Understanding photoreceptor outer segment phagocytosis: Use and utility of RPE cells in culture, Exp. Eye Res. 126 (2014) 51-60.

[58] A. Subrizi, H. Hiidenmaa, T. Ilmarinen, S. Nymark, P. Dubruel, H. Uusitalo, M. Yliperttula, A. Urtti, H. Skottman, Generation of hESC-derived retinal pigment epithelium on biopolymer coated polyimide membranes, Biomaterials. 33 (2012) 80478054.

[59] A. Sorkio, P.J. Porter, K. Juuti-Uusitalo, B.J. Meenan, H. Skottman, G.A. Burke, Surface modified biodegradable electrospun membranes as a carrier for human embryonic stem cell-derived retinal pigment epithelial cells, Tissue Eng. Part A. (2015).

[60] H. Nazari, L. Zhang, D. Zhu, G.J. Chader, P. Falabella, F. Stefanini, T. Rowland, D.O. Clegg, A.H. Kashani, D.R. Hinton, M.S. Humayun, Stem cell based therapies for agerelated macular degeneration: The promises and the challenges, Prog. Retin. Eye Res. 48 (2015) 1-39.

[61] K. Juuti-Uusitalo, H. Vaajasaari, T. Ryhänen, S. Narkilahti, R. Suuronen, E. Mannermaa, K. Kaarniranta, H. Skottman, Efflux protein expression in human stem cell-derived retinal pigment epithelial cells., PloS One. 7 (2012) e30089-e30089. 